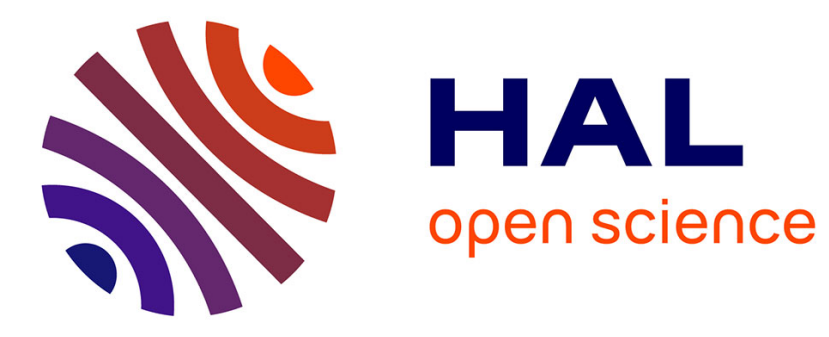

\title{
Fault prognostic of bearings by using support vector data description.
}

Tarak Benkedjouh, Kamal Medjaher, Noureddine Zerhouni, Saïd Rechak

\section{To cite this version:}

Tarak Benkedjouh, Kamal Medjaher, Noureddine Zerhouni, Saïd Rechak. Fault prognostic of bearings by using support vector data description.. IEEE Conference on Prognostics and Health Management, PHM'12., Jun 2012, Denver, United States. pp.1-7, 10.1109/ICPHM.2012.6299511 . hal-00805665

\section{HAL Id: hal-00805665 https://hal.science/hal-00805665}

Submitted on 28 Mar 2013

HAL is a multi-disciplinary open access archive for the deposit and dissemination of scientific research documents, whether they are published or not. The documents may come from teaching and research institutions in France or abroad, or from public or private research centers.
L'archive ouverte pluridisciplinaire HAL, est destinée au dépôt et à la diffusion de documents scientifiques de niveau recherche, publiés ou non, émanant des établissements d'enseignement et de recherche français ou étrangers, des laboratoires publics ou privés. 


\section{Fault Prognostic of Bearings by using Support Vector Data Description}

\author{
T. Benkedjouh \\ EMP, Laboratoire de Mécanique \\ des Structures (LMS), \\ Bordj El Bahri, Algiers, Algeria.
}

\author{
K. Medjaher, N. Zerhouni \\ FEMTO-ST Institute, \\ AS2M department, \\ UMR CNRS 6174 - UFC / \\ ENSMM / UTBM, \\ 25000 Besançon, France. \\ Email: kamal.medjaher@ens2m.fr
}

\author{
S. Rechak \\ ENSP, Laboratoire Génie Mécanique, \\ El-Harrach, Algiers, Algeria.
}

\begin{abstract}
This paper presents a method for fault prognostic of bearings based on Principal Component Analysis (PCA) and Support Vector Data Description (SVDD). The purpose of the paper is to transform the monitoring vibration signals into features that can be used to track the health condition of bearings and to estimate their remaining useful life. PCA is used to reduce the dimensionality of original vibration features by removing the redundant ones. SVDD is a pattern recognition method based on structural risk minimization principles. In this contribution, the SVDD is used to fit the trained data to a hypersphere such that its radius can be used as a health indicator. The proposed method is then applied on real bearing degradation performed on an accelerated life test. The experimental results show that the health indicator reflects the bearing's degradation.
\end{abstract}

Index Terms-Diagnostic, Prognostic, Remaining Useful Life, Support Vector Data Description, Feature extraction and reduction, Condition-Based Maintenance.

\section{INTRODUCTION}

The ability to estimate and predict a machine's failure is primordial as this allows anticipating the failure and leads to increase the availability, reliability and security while reducing the maintenance costs. Traditional maintenance of bearings is either "run-to-failure" or scheduled replacement based on bearings' statistical life. This approach is unreliable because of the greatly variant bearing's life as well as of the variable operating conditions in real applications. Therefore, traditional corrective maintenance tends to be replaced by ConditionBased Maintenance (CBM) and Predictive Maintenance (PM). In a CBM, the machine is continuously monitored in order to evaluate its health state and predict its future one and thus estimate its Remaining Useful Life (RUL) before it fails.

A general CBM architecture is proposed in [1], where seven layers are needed: sensors and data acquisition, signal processing and feature extraction, health assessment and fault detection, fault diagnostic, fault prognostic, decision support and finally, results' presentation on a human-machine interface.

Fault diagnostic can be seen as a problem of pattern recognition, whereas failure prognostic aims at assessing the current health condition of a machine and predicting its future one leading to an estimation of its RUL [5], [6].

The present paper deals with a Support Vector Data De- scription (SVDD) based failure prognostic method. The main contribution dwells in the utilization of the SVDD classifier and on Principal Component Analysis (PCA) features reduction in order to continuously assess the health condition of critical physical components (particularly bearings). SVDD [8] is a technique of mono-class classification, which tries to solve the problem of outliers' detection by distinguishing between a target class and an outliers class. The target class is obtained from learning data that represent the whole life cycle of the physical component. The outliers class results from the following decision rule: the test data are classified as target class only if their distance to the corresponding hypersphere's center is less or equal to the radius of the hypersphere; otherwise they are classified as outliers.

Several research works dealing with the utilization of SVDD have been reported in the recent literature. Examples of applications are face recognition [9], speaker recognition [10] and medical imaging [11]. However, only few of these works deal with health assessment of physical components [8], [12]. The contribution of the present paper consists in a oneclass classification method based on support vector machine [26]. Indeed, the objective is to construct a boundary around the target data by enclosing this latter within a minimum hypersphere. This technique, which aims at enhancing bearing fault diagnostic and prognostic, is developed by fusion of multiple health indicators through SVDD. Firstly, a selection of best features from experimental data is done at the classifier training stage, and then different strategies for features reduction that can maximize the performance of the classifier are studied. The reduction process is realized based on PCA technique. Then, a search for a hypersphere with minimal volume containing most of the mapped training data is performed, where several key variables such as the radius and the center of the hypersphere are used. In this contribution, the radius of the hypersphere is used as a health indicator to reflect the bearings' degradation.

The present paper is organized as follows: section 2 presents the SVDD formalization tool and section 3 describes the steps of the method proposed for failure prognostic of bearings. Section 4 deals with the application of the SVDD method on 
experimental data related to bearings' accelerated degradations and results are presented and discussed. Finally, a conclusion is given in section 5 .

\section{Support Vector DATA DESCRIPTION}

Support Vector Data Description (SVDD) [8], [13] is inspired by the idea of the support vector classifier proposed by Vapnik [14]. SVDD is a method of data domain description, also called one-class classification and has been used for the outlier detection or classification by Tax and Duin [13]. In the SVDD technique, a geometry is made with less number of constraints to describe the data by mapping them to a high-dimensional space where the classification is performed. This leads to a methodology known as kernel in statistics and machine learning and where the purpose is not to find an optimal separating hyperplane, but a spherically shaped boundary around the dataset with minimal volume containing all data. The computing tasks of the SVDD model concern the calculation of the radius and the center of the hypersphere by using the given data samples.

A boundary method for outlier detection based on support vector domain description was presented in [15]. This method attracted many researchers from various applications such as pump failure detection [8], face recognition [9], speaker recognition [10] and medical imaging [11]. Furthermore, SVDD was used for outlier detection to detect uncharacteristic objects from a data set. The boundary of a dataset can be used to detect novel data or outliers. The method can be made flexible by using other kernel functions. In addition, the method is robust against outliers in the training set and is capable of tightening the description by using negative examples.

Let $\left\{x_{i}, i=1,2 \ldots N\right\}$ be the given training dataset with the data space, where $N$ is the number of samples. Let $\alpha$ and $R$ denote the center and the radius of the hypersphere, respectively. The main work in SVDD is then to describe the dataset by using a hypersphere with minimized radius in the feature space. In other words, all the samples should be located in the hypersphere. This main purpose can be formulated as a constrained convex optimization problem by minimizing the function with the constraint condition $\left\|x_{i}-a^{2}\right\| \leq R^{2}(\forall i=1,2, \ldots, N)$. Thus, if outliers appear in the dataset, their corresponding distances to the center of the hypersphere will not be strictly smaller that $R$. To increase the classification's performance, the outliers which have large distances from the center are penalized. To deal with the influence of outliers, slack variable $\xi_{i} \geq 0,(i=1,2, \ldots, N)$ is introduced in the objective function.

The problem of minimizing the radius of the hypersphere can be described by the following quadratic programming with inequality constraints:

$$
\begin{gathered}
\min F\left(R, a, \xi_{i}\right)=R^{2}+C \sum_{i=1}^{N} \xi_{i} \\
\text { s.t. }\left\{\begin{array}{l}
x_{i}-a^{2} \leq R^{2}+\xi_{i}, i=1,2, \ldots, N . \\
\xi_{i} \geq 0, i=1,2, \ldots, N .
\end{array}\right.
\end{gathered}
$$

Where $C$ is a positive constant called penalty factor. This parameter controls the trade-off between the radius of the hypersphere and the testing error. By using the Lagrange multiplier algorithm for Eq.(1), the corresponding Lagrange function becomes:

$$
\begin{aligned}
& L\left(R, a, \alpha_{i}, \beta_{i}, \xi_{i}\right)=R^{2}+C \sum_{i=1}^{N} \xi_{i} \\
& -\sum_{i=1}^{N} \alpha_{i}\left(R^{2}+\xi_{i}-x_{i}-a^{2}\right)-\sum_{i=1}^{N} \beta_{i} \xi_{i}
\end{aligned}
$$

Where $\alpha_{i} \geq 0$ and $\beta_{i} \geq 0$ are Lagrange multipliers. The Lagrange function $L$ should be minimized with respect to $R, a_{i}, \xi$, and maximized with respect to $\alpha_{i}$ and $\beta_{i}$. The limit conditions of Lagrange function $L$ when setting partial derivatives of $R, a_{i}$ and $x_{i}$ and equaling them to zero yields the following constraints:

$$
\frac{\partial L}{\partial R}=0, \frac{\partial L}{\partial a}=0, \frac{\partial L}{\partial \xi_{i}}=0
$$

such that

$$
\begin{gathered}
\sum_{i=1}^{N} \alpha_{i}=1, \\
a=\sum_{i=1}^{N} \alpha_{i} x_{i} \\
C-\alpha_{i}-\beta_{i}=0
\end{gathered}
$$

Note that one can get $0 \leq \alpha_{i} \leq C$ from Eq.(7) because $\alpha_{i} \geq 0$ and $\beta_{i} \geq 0$. Furthermore, when Eqs.(5) to (7) are substituted into the Lagrange function of Eq.(3), the dual form of the Lagrange optimization problem turns into:

$$
\begin{gathered}
\max L=\sum_{i=1}^{N} \alpha_{i}\left(x_{i} . x_{i}\right)-\sum_{i=1}^{N} \sum_{j=1}^{N} \alpha_{i} \alpha_{j}\left(x_{i} . x_{j}\right) \\
\left\{\begin{array}{l}
\sum_{i=1}^{N} \alpha_{i}=1 \\
0 \leq \alpha_{i} \leq C, i=1,2, \ldots, N .
\end{array}\right.
\end{gathered}
$$

Where $x_{i} \cdot x_{j}$ stands for the inner product of $x_{i}$ and $x_{j}$. Usually, the dataset is not ideally distributed in the hypersphere. So, the inner product can be substituted by some kernel function in high-dimensional feature space.

After solving the quadratic programming problem containing the inequality constraints denoted by Eqs.(8) and (9), the parameters of the SVDD model $\left\{\alpha_{i}, \mathrm{i}=1,2 \ldots \mathrm{N}\right\}$ can be estimated. The parameters satisfy the previous conditions given in Eqs.(5) to (7).

Solving the problem of Eq.(10) gives the set $\alpha_{i}$. A training data $x_{i}$ and its corresponding $\alpha_{i}$ satisfy one of the three conditions given in Eqs.(5) to (7).

$$
\left\{\begin{array}{l}
x_{i}-a^{2}<R^{2} \rightarrow \alpha_{\mathrm{i}}=0 \\
x_{i}-a^{2}=R^{2} \rightarrow 0 \leq \alpha_{i} \leq C \\
x_{i}-a^{2}>0 \rightarrow \alpha_{\mathrm{i}}=C
\end{array}\right.
$$

The data with the coefficients $\alpha_{i}>0$ are called the Support Vectors $(S V)$. From the above relations one can see that only 
the SVs are needed in the description of the hypersphere. The hypersphere's center could be calculated by using Eq.(10). The radius $R$ of the hypersphere can be obtained by calculating the distance from its center to any support vector with $0 \leq \alpha_{i} \leq C$, which provides the sparse representation of the domain description.

To determine whether a test data $z$ is within the hypersphere, its distance to the center of the hypersphere has to be calculated. A test data $z$ is accepted when this distance is smaller than the radius, i.e.:

$z-a^{2}=(z . z)-2 \sum_{i=1}^{N} \alpha_{i}\left(z . x_{i}\right)+\sum_{i=1}^{N} \sum_{j=1}^{N} \alpha_{i} \alpha_{j}\left(x_{i} . x_{j}\right) \leq R^{2}$

By definition, $R^{2}$ is the distance from the center of the hypersphere $\alpha$ to any of the support vectors on the boundary. Thus, support vectors which fall outside the description $\left(\alpha_{i}=C\right)$ are excluded. Therefore:

$$
R^{2}=\left(x_{k} . x_{k}\right)-2 \sum_{i=1}^{N} \alpha_{i}\left(x_{i} . x_{k}\right)+\sum_{i=1}^{N} \sum_{j=1}^{N} \alpha_{i} \alpha_{j}\left(x_{i} . x_{j}\right),
$$

for any $x_{k} \in S V<C$, the set of support vectors which have $\alpha_{k}<C$. So,

$$
\sum_{i=1}^{N} \sum_{j=1}^{N} \alpha_{i} \alpha_{j}\left(x_{i} . x_{j}\right)=\text { const }
$$

Then,

$$
R=\sqrt{f\left(x_{s}\right)}
$$

where $x_{s}$ can be an arbitrary support data. For a test data $z$, its distance to the center $\alpha$ is given by the following expression:

$$
\Delta=\sqrt{f(z)}-R
$$

So, the state of $z$ (for which the distance to the center is less or greater than $R$ ) can be decided according to the following criterion:

$$
\left\{\begin{array}{l}
\Delta \leq 0 \text { is target } \\
\Delta>0 \text { is outlier }
\end{array}\right.
$$

The SVDD method can be made more flexible by using kernel functions [8]. An ideal kernel function would map the target data onto a bounded spherically shaped area in the feature space and outlier objects outside this area. Many kernel functions have been proposed for the support vector classifier. However, not all of them are equally useful for the SVDD. Indeed, it has been shown in [15] that the Gaussian kernel:

$$
k(x, y)=\exp \left(\frac{\mathrm{x}-\mathrm{y}}{\sigma^{2}}\right)^{2}
$$

where $\sigma$ is a width parameter (also called extension constant) is more efficient than other kernel functions, such as polynomial function, sigmoid function, etc. This kernel is independent of the position of the data set with respect to the original data and utilizes only the distances between data. By selecting a Gaussian kernel, one gets the following equation:

$$
f(z)=1-2 \sum_{i=1}^{N} \alpha_{i} K\left(z \cdot x_{i}\right)+\sum_{i=1}^{N} \sum_{j=1}^{N} \alpha_{i} \alpha_{j}\left(x_{i} . x_{j}\right)
$$

Figure 1 shows a sketch map of a hypersphere boundary

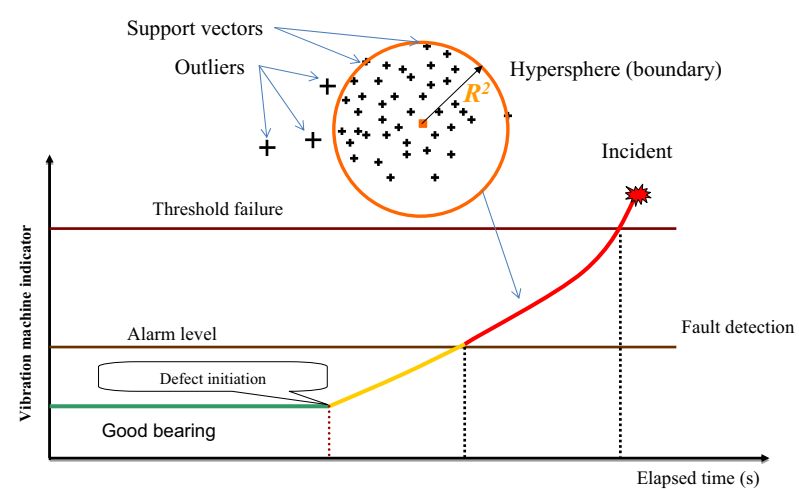

Fig. 1. Sketch map of SVDD of machine life.

defined by a support vector data description. The plus (+) samples inside the sphere are targets, those $(+)$ outside the sphere are outliers and the samples on the boundary are support vectors. Both target and outlier samples on the wrong side of the boundary are associated with slack variables in order to deal with testing errors.

\section{SVDD FOR BEARINGS' FAILURE PROGNOSTIC}

The present section deals with the proposed method for bearing's health assessment and prognostic based on the use of SVDD. The steps of the method are shown in Fig. 2.

The main idea of the proposed contribution relies on the transformation of the raw monitoring data, about the bearing's degradation, to relevant models that can be used to represent the behavior of the degradation, continuously estimate the heath state of the bearing, predict its future one and finally calculate its RUL value.

For the sake of clarity and simplicity, the two following steps of Fig. 2 are explained: feature extraction and reduction and health estimation and RUL estimation method. The SVVD approach being presented in section II.

\section{A. Features extraction and reduction}

In the current contribution, the raw monitoring vibration signals are first preprocessed (filtering, removing of outliers, etc.) before extracting original features. The features can be of three types: temporal, frequency and time-frequency. The number of generated features is then reduced to a small number, which is finally used for bearing's health assessment and RUL estimation. Indeed, the concept of feature extraction used to accurately assess the bearing degradation is a critical step in the learning phase of the health assessment model as well as in the on-line phase for RUL estimation. In this paper, PCA technique is used to select and reduce the features 


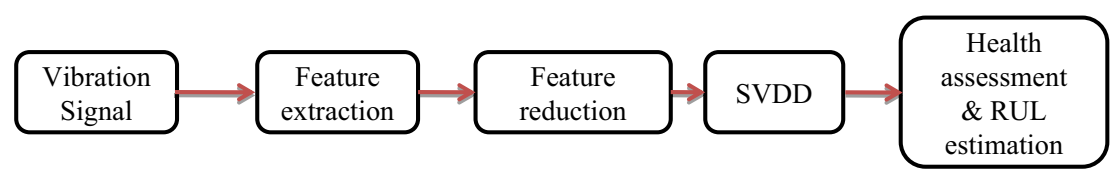

Fig. 2. Main steps of the SVDD failure diagnostic and prognostic.

extracted from raw signals by considering $97 \%$ of selection to keep most variance information. PCA is a statistical technique widely used in the pattern recognition community as a method of feature reduction [16]. This technique is based on an orthogonal decomposition of the covariance matrix of the process variables along directions that explain the maximum variation of the data. This is carried out by performing a rotation of the data in the feature space, and creating a new set of axes (dimensions), which maximizes the variance in each dimension as much as possible. After the transformation phase, the data is ordered with maximum variance and this yields to the first few dimensions.

In this work, 17 features were initially extracted (root mean square, kurtosis, etc.), then this number is reduced to only 3.

\section{B. Bearing's health assessment and RUL estimation}

The diagram of the proposed SVDD method for bearing's health assessment and failure prognostic is given in Fig. 3. The bearing's health assessment and RUL estimation is done in two phases: a first phase to build the SVDD model (learn the radius and the center of the hypersphere), and a second phase to continuously assess the bearing's condition and to calculate its RUL. During the first phase, the set of reduced features is used (the values of the features at each time are fed to the learning algorithm). In the second phase, the learned model is used on a test bearing with new reduced features to determine its current condition and to calculate its RUL.

The RUL calculation is based on the evolution of the severity

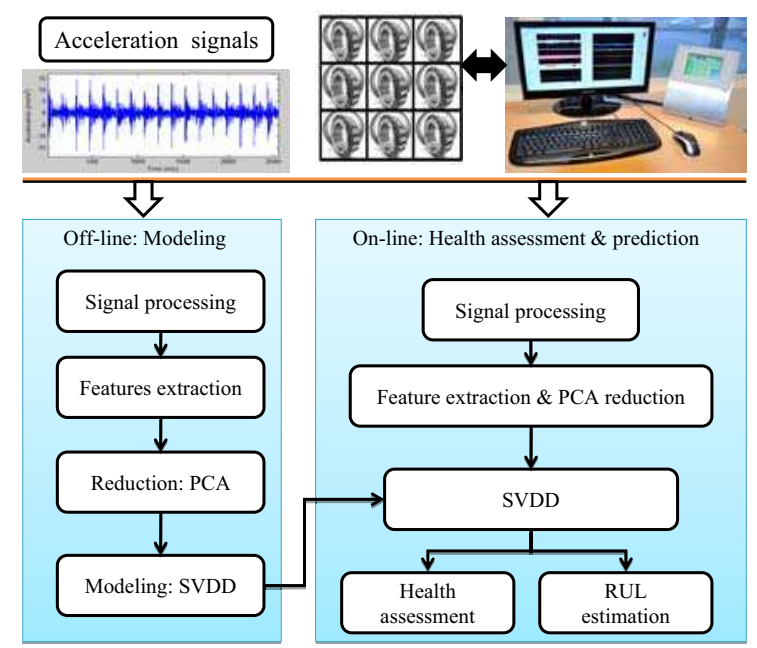

Fig. 3. System framework for bearing performance degradation assessment.

of the degradation. Figure 4 illustrates how this is done.
The right part of figure 4 corresponds to the time evolution of the SVDD hypersphere's radius, which is related to the evolution of the degradation. Thus, by observing the trend curve of the severity (right side of figure 4) and by knowing the accepted threshold of the bearing's failure, one can determine the value of the RUL. Mathematically speaking, let $R(t)$ be the corresponding analytical function of the severity evolution and $\epsilon$ be the predefined threshold from which the bearing is out of service. Then, the estimated value of RUL at time $t_{0}$ can be obtained by using the following expression (the uncertainty of RUL prediction is not taken into account in this formula):

$$
R U L=R^{-1}(\varepsilon)-t_{0}
$$

\section{Application On PRONOSTIA}

\section{A. Description of the experimental setup}

An accelerated bearing life test platform called PRONOSTIA (Fig. 5) is used in this section to verify the prognostic SVDD method proposed in section III. PRONOSTIA is a laboratory experimental platform dedicated to test, verify and validate developed methods related to bearing health assessment, diagnostic and prognostic. In the following of the paper, a set of three experiments consisting of three degraded bearings has been utilized. The three data sets have the following time durations: 6 hours and 50 minutes, 6 hours and 48 minutes and finally 6 hours and 16 minutes.

PRONOSTIA is composed of two main parts: a first part related to the speed variation and a second part dedicated to load profiles generation. The speed variation part is composed of a synchronous motor, a shaft, a set of bearings and a speed controller. The second part is composed of a hydraulic jack connected to a lever arm used to create different loads on the tested bearing mounted on the platform. A pair of ball bearings is mounted on one end of the shaft to serve as the guide bearings and a NSK6307DU roller ball bearing is mounted on the other end to serve as the test bearing. Two high frequency accelerometers are mounted horizontally and vertically on the housing of the tested roller bearing to pick up the horizontal and the vertical accelerations. The sampling frequency of the data acquisition card is set to $25600 \mathrm{~Hz}$ and the vibration data provided by the two accelerometers are recorded every 1 second. In the following experiments the rotation speed of the motor's shaft is kept constant at $1800 \mathrm{rpm}$. A radial load of $400 \mathrm{kN}$ is applied on the shaft and the bearing by a hydraulic jack pressure mechanism.

\section{B. Experimental results}

1) Extracted features: figure 6 shows the kurtosis of the vibration signal. From this figure, one can observe two dif- 

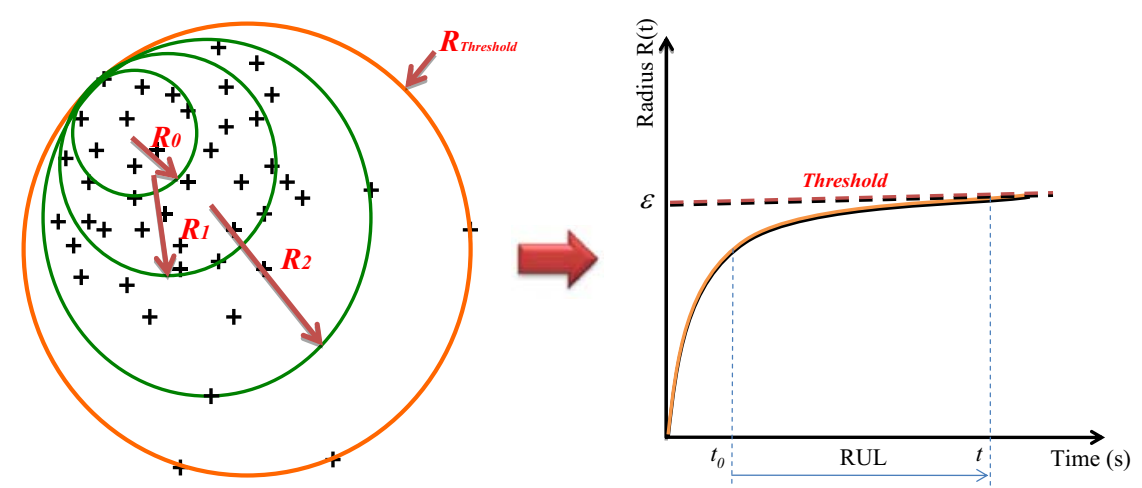

Fig. 4. Severity evolution of the degradation and RUL estimation.

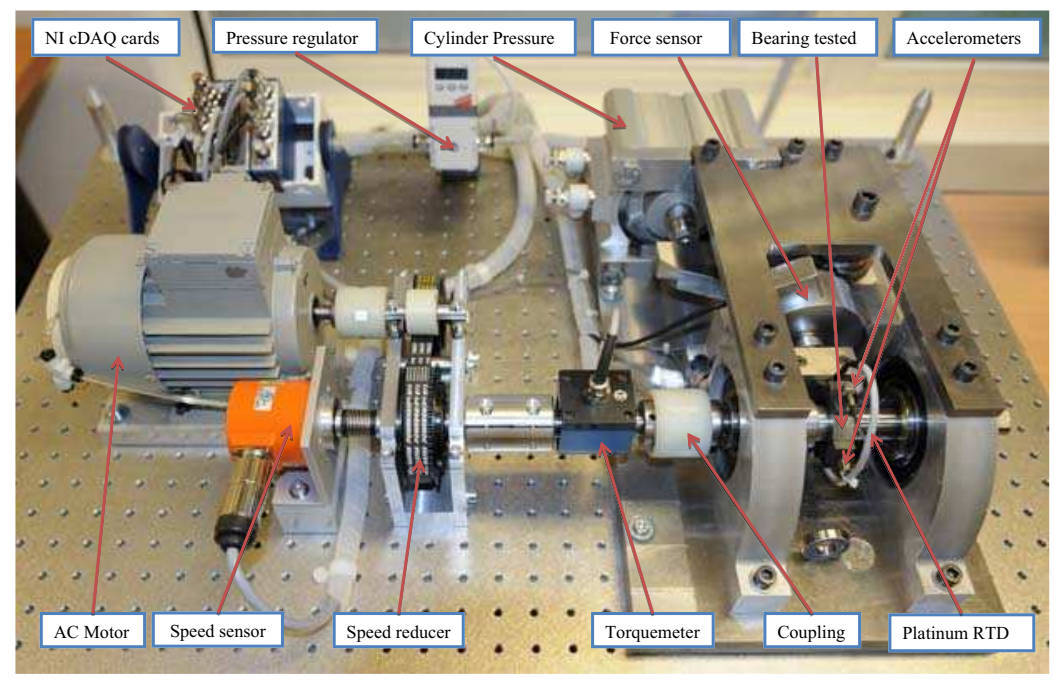

Fig. 5. Overview of PRONOSTIA platform.

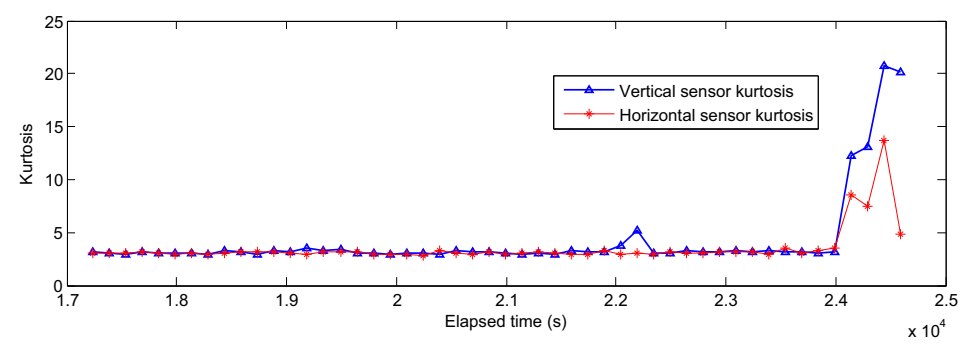

Fig. 6. Kurtosis value for bearing 1 in horizontal and vertical directions.

ferent regions. The first region is flat representing the normal bearing operation. The second region is characterized by a fluctuating signal with an increasing trend. Generally, vibration signals of healthy bearings are Gaussian in distribution, in any value of speed and load. Therefore the value of the kurtosis is close to three for the vibration signals of healthy bearings. The appearance of a fatigue crack or a spall on the bearing surfaces results in kurtosis values that are greater than three. However, if the damage increases the kurtosis values come back to three.
2) SVDD based health assessment and RUL estimation: in this study, healthy bearings are run until they are completely failed. Then, the data from the whole life of the bearings are collected. From each tested degradation, $50 \%$ of the monitoring data are used for training and the remaining 50\% are used to test the models and to estimate the health condition of the bearing.

The experimental results presented in this section are conducted in two categories and the SVDD model is trained by using the SVDD toolbox described in [17]. The first set of 


\begin{tabular}{|l|c|c|c|c|}
\hline kernel Test & Train time(s) & Precision (\%) & Train Test time $(\mathrm{s})$ & Precision (\%) \\
\hline Polynomial SVDD $(\mathrm{d}=5)$ & 9.73 & 89.23 & 1.52 & 88.45 \\
\hline Gaussian SVDD & 13.58 & 93.45 & 1.78 & 92.78 \\
\hline Sigmoid SVDD & 11.96 & 88.12 & 2.14 & 86.47 \\
\hline
\end{tabular}

Fig. 7. Recognition results.

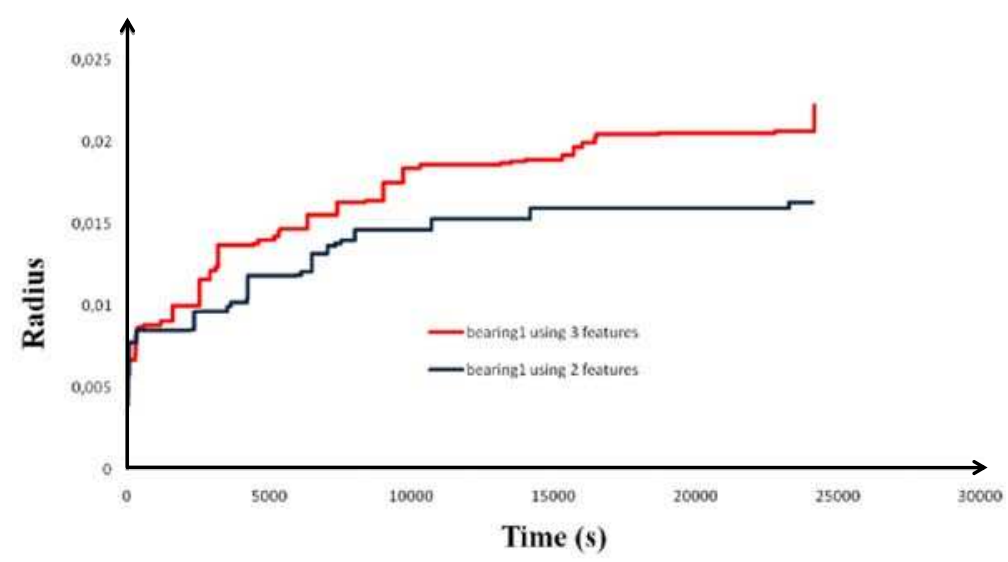

Fig. 8. Radius evolution of minimum enclosing ball using 2 and 3 features.

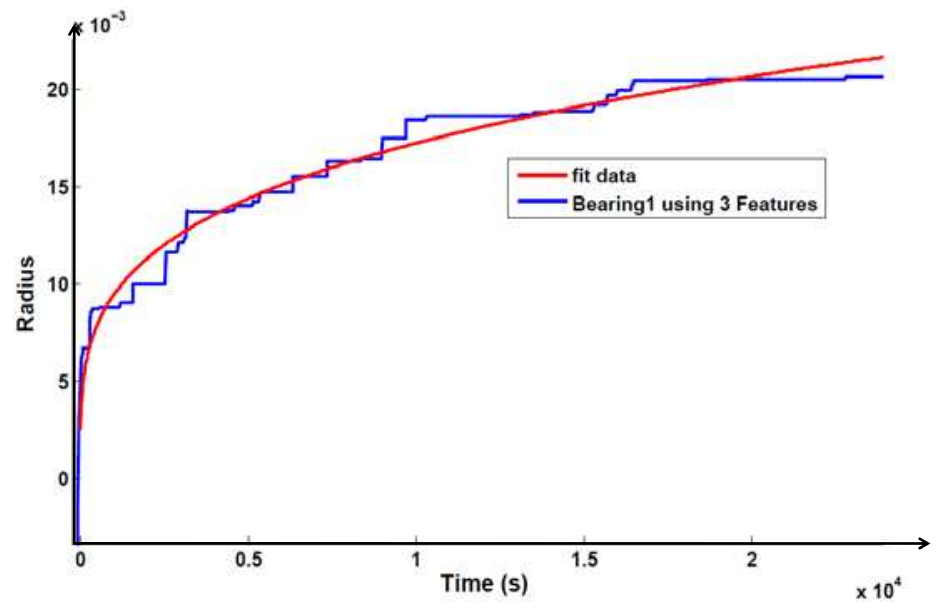

Fig. 9. Curve fitting data for RUL estimation.

results is devoted for the fault detection of bearing faults and for evaluating the performance of different kernel functions for the training dataset. In the second set, the severity of the bearing's defect is tracked by using a minimum enclosing ball to predict its health state and estimate its RUL value. For this purpose, the radius of the hypersphere generated by the SVDD is used as a health indicator. Furthermore, an appropriate degradation threshold indicating the failure of the bearing is defined.

The kernel function used in this work is Gaussian, where the parameter $\sigma$ is set between 0.01 and 0.1 [15]. The results of the Gaussian kernel function are compared to those obtained by using polynomial and sigmoid kernels. Figure 7 compares the classification error and the test time for various classifications of different kernel functions.
After the extraction of features from the raw signals which have the potential to be correlated with the bearing's degradation, a feature selection step is conducted. Then, the selected features are used as inputs of the SVDD method to estimate the health condition of the bearing. Note that the accuracy of the algorithm is dependent on the quality of features being used for training. Firstly, 17 original features are generated from the training data and then PCA is implemented on the features to reduce their number.

In the following tests, a Gaussian kernel is used to assess the health condition of the bearings and to estimate their RUL. Thus, a search for an optimal combination of kernel width $\sigma$ and number of principal components is performed. To speed up the search, any eigenvector whose corresponding eigenvalue is smaller than $10^{-4}$ is discarded. Moreover, in 


\begin{tabular}{|l|c|l|c|c|c|}
\hline Test & Number of FE & Exponential regression & SSE & R-square & RMSE \\
\hline \multirow{2}{*}{ Test1 (6h50) } & 2 Features & $R(t)=0.001918 * t^{0.2162}$ & 0.012990 & 0.9213 & 0.0007343 \\
\cline { 2 - 6 } & 3 Features & $R(t)=0.001549 * t^{0.2615}$ & 0.009656 & 0.9709 & 0.0006321 \\
\hline \multirow{2}{*}{ Test2 (6h48) } & 2 Features & $R(t)=0.004025 * t^{0.1978}$ & 0.000618 & 0.9405 & 0.0006135 \\
\cline { 2 - 6 } & 3 Features & $R(t)=0.003675 * t^{0.2479}$ & 0.001915 & 0.9812 & 0.0005142 \\
\hline \multirow{2}{*}{ Test3 (6h16) } & 2 Features & $R(t)=0.000899 * t^{0.2003}$ & $6.249 \mathrm{e}-5$ & 0.8976 & 0.0001918 \\
\cline { 2 - 6 } & 3 Features & $R(x)=0.002061 * t^{0.0606}$ & 0.013200 & 0.9414 & 0.0019560 \\
\hline
\end{tabular}

Fig. 10. Radius regression functions.

order to improve the robustness of the proposed SVDD method for health assessment and RUL estimation, several simulations have been carried out by modifying the number of features based on PCA. Figure 8 shows the radius evolution of the hypersphere for 2 and 3 features.

In the table given in Fig. 10, which represents the radius of minimum enclosing ball, one can observe that the health indicator evolution is more better than the kurtosis shown in figure (6). Furthermore, this fitting is better if only three features selected with maximum variance are used after reduction by PCA. This dimension space of features reflects the effect of power regression error to follow-up the severity of the defect and estimate the RUL. The regression equations are shown in the table of Fig. 10 where a power regression model is used in order to estimate the RUL. The objective is to apply the best power fit of the radius on the degradation signal. Thus, the RUL can be easily calculated by using the regression curve shown in Fig. 9.

The validation of the experimental results are shown in the table of Fig. 10. The sum square error (SSE), the $R$ square and the root mean square error (RMSE) are calculated. One can see that these errors decrease when the number of features selected after reduction increases. However, this increases the runtime of training data.

\section{CONCLUSION}

A method based on PCA and SVDD for health assessment and life prediction of bearings is proposed in this paper. This study investigates the efficiency of SVDD for estimating the remaining useful life by analyzing complex and nonlinear data of bearings' life cycle. The study also explores the advantages of the SVDD model for the health assessment of bearings. The model's output was obtained and compared to the experimentally measured data by using a regression function.

The developed method can be considered within the framework of condition based maintenance and predictive maintenance. This new approach aimed to make the SVDD boundary closely fit the contour of the target data. The simulations have shown that using PCA for features reduction gives better results. Moreover, a combination of PCA and SVDD has been used for intelligent fault prognostic. The experimental results of bearings' degradations have shown that the proposed method can reduce the effect of outliers and yield higher classification rate. The proposed SVDD method can be seen as a way of automatically performing health assessment, failure prediction and RUL estimation for bearings. Finally, this method can be applied on other physical components, other than bearings, provided that monitoring data which reflect the behavior of the component's degradation are available.

\section{REFERENCES}

[1] M. Lebold, M. Thurston, Open standards for condition-based maintenance and prognostic systems, in: Proc. 5th Maintenance and Reliability Conference (MARCON), 2001.

[2] A. K. Jardine, D. Lin, D. Banjevic, A review on machinery diagnostics and prognostics implementing condition-based maintenance, Mechanical Systems and Signal Processing 20 (7) (2006) 1483 - 1510.

[3] A. Heng, A. C. Tan, J. Mathew, N. Montgomery, D. Banjevic, A. K. Jardine, Intelligent condition-based prediction of machinery reliability, Mechanical Systems and Signal Processing 23 (5) (2009) 1600 - 1614.

[4] D. Tax, R. Duin, Support vector data description, Machine Learning 54 (1) (2004) 45-66.

[5] S. W. Lee, J. Park, S. W. Lee, Low resolution face recognition based on support vector data description, Pattern Recognition 39 (9) (2006) 1809-1812.

[6] X. Dong, H. Zhao, Z. Wan, Support vector domain description for speaker recognition, in: Proceedings of the 2001 IEEE Signal Processing Society Workshop, 2001, pp. 481-488.

[7] K. Sjöstrand, M. S. Hansen, H. B. Larsson, R. Larsen, A path algorithm for the support vector domain description and its application to medical imaging, Med. Image Anal. 11 (2007) 417-428.

[8] L. Lingjun, H. Jie, H. Wei, D. Xinmin, H. Zhengjia, Condition evaluation for mechanical equipment by means of support vector data description, Chinese Journal of Mechanical Science and Technology 24 (12) (2005) 1426-1429.

[9] D. Tax, R. Duin, Support vector domain description, Pattern Recognition Letters 20 (11-13) (1999) 1191-1199.

[10] V. Vapnik, The Nature of Statistical Learning Theory, Springer, New York, 1995.

[11] S. Guo, L. Chen, J. Tsai, A boundary method for outlier detection based on support vector domain description, Pattern Recognition 42 (2009) 77-83.

[12] A. Malhi, R. Gao, PCA-based feature selection scheme for machine defect classification, IEEE Transactions on Instrumentation and Measurement 53 (2004) 1517-1525.

[13] Ddtool, in: http://www-ict.ewi.tudelft.nl/_davidt/dd_tools.html. 